\title{
En torno a la evolución de la investigación en Contabilidad Social y Medioambiental
}

\author{
On the evolution of Social and Environmental Accounting Research
}

\author{
Carlos Larrinaga González \\ Autor Invitado de Honor
}

\section{ESPAÑA}

\begin{abstract}
Resumen: La intención de este documento es caracterizar la evolución de la investigación en contabilidad social y medioambiental, para ofrecer una visión de conjunto -sin ser sistemática ni exhaustiva- sobre este campo académico. Inicialmente se analiza la evolución de este campo de investigación a la luz de diferentes perspectivas teóricas, como el enfoque normativo, el marco conceptual y el paradigma de la utilidad para la toma de decisiones. Luego se exponen las teorías descriptivas aplicadas al reporte de información social y medioambiental de las empresas desde los enfoques de la teoría de los grupos de interés, la teoría de la legitimidad y la teoría institucional. El texto hace precisiones conceptuales que favorecen la comprensión de esta clasificación y advierte las posibilidades de otros enfoques más progresistas para guiar el estudio de la temática.
\end{abstract}

Palabras clave: contabilidad social y ambiental; investigación contable; contabilidad para la sostenibilidad; heterodoxia contable.

Abstract: The aim of this document is to characterize the evolution of social and environmental accounting research, to offer an overall vision -without being systematic or exhaustive- about this academic field. Initially, the evolution of this field of research is analyzed in the light of different theoretical perspectives, such as the normative approach, the conceptual framework and the decisionusefulness paradigm. Then, the paper discusses the application of descriptive theories to the study of corporate social and environmental reporting, including stakeholder theory, legitimacy theory and institutional theory. The text elaborates on conceptual issues to facilitate the understanding of this classification and notes the existence of more progressive approaches to drive the study of the subject.

Keywords: social and environmental accounting; accounting research; accounting for sustainability; accounting heterodoxy.

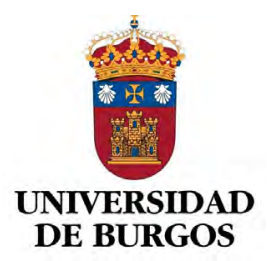

Carlos Larrinaga González. Licenciado en Ciencias Económicas y Empresariales, y Doctor en Administración de Empresas por la Universidad de Sevilla; ha sido profesor visitante en las universidades de Glasgow, St. Andrews y Northwestern; es autor de numerosas publicaciones en los campos de la Gestión y Contabilidad Social y Ambiental; miembro de comités editoriales y científicos de varias revistas especializadas en Europa y América, y es miembro de diversas organizaciones académicas internacionales, entre ellas, el Comité Ejecutivo de la European Accounting Association. Actualmente es profesor investigador Catedrático de la Universidad de Burgos (España) y asociado internacional del Centro de Investigación en Contabilidad Social y Medioambiental (CSEAR) de la Universidad de St. Andrews (Escocia).

Contacto: carlos.larrinaga@ubu.es 


\section{Em torno a evoluçao da pesquisa em Contabilidade Social e Ambiental}

Resumo: A intenção deste documento é caracterizar a evolução da pesquisa em contabilidade social e ambiental, para oferecer uma visão geral -sem ser sistemática ou exaustiva- sobre esse campo acadêmico. Inicialmente, a evolução deste campo de pesquisa é analisada à luz de diferentes perspectivas teóricas, como a abordagem normativa, a estrutura conceitual e o paradigma de utilidade para a tomada de decisões. Em seguida, as teorias descritivas aplicadas ao relatório de informações sociais e ambientais das empresas são expostas das abordagens da teoria dos grupos de interesse, a teoria da legitimidade e a teoria institucional. $O$ texto faz precisões conceituais que favorecem a compreensão desta classificação e avisa as possibilidades de outras abordagens mais progressivas para orientar o estudo do assunto

Palavras-chave: contabilidade social e ambiental; pesquisa contábil; contabilidade pela sustentabilidade; heterodoxia contábil.

Para comenzar, quiero agradeceral profesor HéctorJoséSarmiento y al Consejo Editorial de la revista Teuken-Bidikay la deferencia que han tenido al invitarme a participar en este número. Para tratar de dar respuesta a tan considerado ofrecimiento, me propongo abordar una revisión personal del desarrollo histórico de la investigación en contabilidad social y medioambiental, haciendo mención a la vigencia actual de los diferentes desarrollos teóricos.

uiero pedir disculpas a aquellos lectores que busquen en este artículo una revisión sistemática de la investigación en contabilidad social y medioambiental, pues me referiré exclusivamente a algunos estudios que permitan ilustrar el devenir histórico de la investigación en esta área. Aquellos interesados en revisiones sistemáticas de la literatura pueden acudir, por ejemplo, a Hahn y Kühnen (2013). También pido excusas a aquellos lectores que busquen ideas nuevas, pues muchas de las ideas aquí expuestas se inspiran en Larrinaga (1999), Deegan y Unerman (2011) y Gómez y Ospina (2009), entre otros.

En esta revisión personal trazaré un paralelismo entre los diferentes paradigmas de investigación en contabilidad y el desarrollo histórico de la contabilidad social y medioambiental. Dadas las limitaciones de espacio de este artículo, me limitaré a la revisión de las perspectivas más convencionales de la investigación en contabilidad y de las teorías descriptivas. Se trata así de un punto de partida que permita articular el estado actual de la contabilidad social y medioambiental $y$, de este modo, dejar a otros autores proseguir en el análisis de las perspectivas más 
constructivistas, interdisciplinares o críticas (Gómez y Ospina, 2009) que tan interesantes frutos están dando en la investigación en contabilidad social y medioambiental.

\section{Perspectivas convencionales de la contabilidad social y medioambiental}

Dentro de las perspectivas convencionales de la investigación en contabilidad social y medioambiental distinguiré entre sus inicios normativos, la investigación en torno al marco conceptual y el paradigma de utilidad.

\section{Inicios normativos de la contabilidad social y medioambiental}

Más allá de la descripción de prácticas y de normas contables, podemos afirmar que el inicio de la investigación económica sobre la contabilidad tuvo lugar cuando diferentes estudiosos, entre los que cabe destacar a Edwards y Bell o McSweeney cuestionaron el rigor de la valoración a costes históricos, habitualmente utilizada en contabilidad, en situaciones de variación del nivel general y específico de los precios. Estos autores cuestionan la relevancia de la información financiera basada en costes históricos, la propiedad aditiva de valores obtenidos en diferentes momentos y la eventual descapitalización de las empresas, producida por la inflación del beneficio (Deegan y Unerman, 2011).

Aunque esta corriente de investigación normativa esté hoy en declive, hay que constatar que produjo ideas que están hoy presentes en la práctica y las normas contables. Pero lo más interesante de esta perspectiva de investigación, desde mi punto de vista, es la idea de que el beneficio calculado de acuerdo con normas contables puede ser inconsistente con su esencia económica. Desde este concepto solo hay un paso a la consideración de un aspecto económico excluido del cálculo del beneficio contable; me refiero a las externalidades. En el contexto de la actividad de las organizaciones, la externalidad es el coste de los efectos sociales y medioambientales de las empresas que no soportan éstas, sino terceros que no perciben beneficios. Por ejemplo, las emisiones de dióxido de carbono y otros gases causantes del efecto invernadero, producirán un coste que soportarán individuos que no obtienen beneficio a cambio. En consecuencia, se puede afirmar que el beneficio de la empresa emisora de dióxido de carbono, calculado contablemente, es inconsistente con el beneficio económico de la empresa, desde un punto de vista social.

En los años setenta del pasado siglo surgieron diferentes estudios (Estes, 1972; Ramanathan, 1976) que proponían modificaciones en el modelo contable para identificar, valorar e incluir costes y beneficios 
medioambientales. Se trata, en definitiva, de encontrar un "beneficio verdadero", utilizando el término de Belkaoui (2004). Esta idea ha tenido continuidad en investigaciones realizadas durante los últimos años, poniendo de manifiesto la consistencia teórica de estos desarrollos conceptuales, al tiempo que su dificultad práctica para llevarlas a cabo en diferentes empresas (Antheaume, 2004; Bebbington y Gray, 2001). A título de ejemplo, Antheaume (2004) estudió un proceso industrial en Francia, derivando externalidades que iban desde 1,5 hasta más de 15.000 euros por unidad de producto, dependiendo de los métodos e hipótesis de valoración seguidos. A pesar de estas dificultades, algunas empresas han venido elaborando cuentas en las que se integran estimaciones de sus externalidades. Un ejemplo de ello es la compañía de ferrocarriles española RENFE, que estima cada año el importe del ahorro de externalidades proporcionado por la utilización del tren en lugar de otros medios de transporte (www.renfe.com). En el ejercicio 2015 este ahorro se estimó en 1.703 millones de euros. En algunos lugares hemos defendido la oportunidad de seguir investigando en estas cuestiones (Bebbington y Larrinaga, 2014).

\section{Marco conceptual}

Me referiré también a una segunda línea de investigación normativa que ha dado lugar al marco conceptual de la contabilidad financiera (Pina Martínez, 1991). El marco conceptual consiste en un sistema coherente de objetivos y fundamentos interrelacionados que se derivan a partir de ciertos postulados a través de un método lógico deductivo, de forma que se puedan obtener normas contables consistentes, en lugar de una formulación ad hoc de normas contables.

Estas ideas también germinaron en el terreno de la contabilidad social y medioambiental, mediante las ideas, por ejemplo, de Rob Gray y sus colegas (Gray, 1992, 2002; Gray, Owen y Maunders, 1987) sobre la rendición de cuentas (accountability). En el terreno de las propuestas normativas también se trató desde los años noventa del pasado siglo, de proporcionar coherencia a los informes medioambientales primero ( $\mathrm{FEE}$, 2000) y a los informes de sostenibilidad después (GRI, 2016), mediante un marco conceptual adaptado a aspectos medioambientales y de desarrollo sostenible. Por ejemplo, el marco (conceptual) de GRI establece los principios e indicadores de desempeño que las organizaciones deben utilizar para medir e informar sobre su desempeño económico, ambiental y social.

La piedra angular de este marco son las sucesivas guías para la elaboración de Memorias de Sostenibilidad, la última de las cuales se publicó en 2016. 
La guía GRI se ha convertido en un referente ineludible para las grandes corporaciones transnacionales y muchas otras organizaciones que quieren elaborar este tipo de informes. Estudios recientes (Ehnert, Parsa, Roper, Wagner y Muller-Camen, 2016; Junior, Best y Cotter, 2014) han encontrado que casi el $100 \%$ de las mayores 250 empresas del mundo tiene algún tipo de actividad en la publicación de este tipo de informes. Las encuestas de KPMG (2017) extienden este grado de aceptación de los informes de responsabilidad social a las mayores empresas de muchos países. Así, este informe afirma que más del $80 \%$ de las 100 mayores empresas de Colombia divulgan este tipo de información. Esto ha llevado a algunos autores a afirmar que los informes de sostenibilidad se han institucionalizado (Cho, Laine, Roberts y Rodrigue, 2015).

Sin embargo, la literatura está lejos de coincidir con los optimistas pronósticos de KPMG. Es en este sentido que se ha desarrollado una influyente corriente de investigación en contabilidad socialy ambiental que trata de demostrar la brecha existente entre la revelación de información por parte de las empresas y su desempeño real, contradiciéndose en cierto modo el supuesto beneficio de la información de sostenibilidad (Boiral, 2013; Cho, Guidry, Hageman y Patten, 2012; Gray, 2010) (ver más adelante también en relación con las teorías descriptivas). Estas conclusiones son de una importancia capital si consideramos la jerarquía de objetivos y principios contables (materialidad o fiabilidad, entre otros) que deben regir la elaboración de los informes de sostenibilidad. Así, Gray (2010) afirmaba que "Cada vez está más establecido en la literatura que la mayor parte de la información empresarial sobre sostenibilidad y de su actividad representativa en torno a la sostenibilidad en realidad tienen poco o nada que ver con la sostenibilidad" (p. 48).

Esta corriente de investigación, que podría denominarse "crítica funcional" de la contabilidad social y medioambiental, es un área de trabajo relevante en la que se necesitan más trabajos empíricos que pongan de manifiesto la deficiente aplicación de las características cualitativas de la información a la comunicación corporativa sobre responsabilidad social y sostenibilidad, y planteen posibilidades para su mejora. Ya hay mucha literatura respecto de los informes de sostenibilidad social y sostenibilidad, particularmente en los países anglosajones. Pero la literatura internacional está en gran medida huérfana de evidencia sobre los desarrollos que se producen en áreas geográficas tan importantes como Latinoamérica. También hay nuevas iniciativas que suscitan las mismas (si no más) dudas sobre el desarrollo de su marco conceptual, como las del Consejo Internacional de Reporting Integrado (IIRC por sus siglas en inglés) o las del Consejo de Normas de Información sobre el Clima (CDSB por sus siglas en inglés). 
Sin embargo, se hace necesario que la crítica funcional sobre la contabilidad social conjugue el análisis conceptual propio de la contabilidad, con estudios empíricos que pongan de manifiesto las prácticas reales de las empresas y con teorías más amplias que permitan dar mayor alcance al resultado de las investigaciones. Si me permiten decirlo así, la investigación en esta área no debe contentarse con repetir información trivial, como el enunciado de los principios contables, ni tampoco debe solazarse por los caminos de la especulación florida, sino que debe tratar de ir más allá, conectando de forma rigurosa y analítica con la literatura previa y siempre con la vista puesta en la contribución de la investigación.

\section{Paradigma de utilidad}

En los manuales de teoría de la contabilidad se destaca que el inicio de la investigación empírica de mercados en contabilidad estuvo asociado con el desarrollo del paradigma de utilidad, de acuerdo con el cual el criterio para dirimir entre diferentes prácticas y normas contables no es su lógica normativa económica, sino su valor económico reflejado en precios de mercado. El criterio es la relevancia valorativa de la información contable en el mercado de capitales. Un trabajo precursor de esta línea de investigación fue el de Ball y Brown (1968), en el que se examina el impacto de cambios en el resultado empresarial en el precio de las acciones.

La traducción de estas ideas al terreno de la contabilidad social y medioambiental consiste en que la información social y medioambiental es útil si tiene relevancia valorativa, es decir, si afecta a magnitudes de mercado tales como el riesgo (Spicer, 1978; Trotman y Bradley, 1981) o el precio de los valores (Cormier, Magnan y Morard, 1993; Freedman y Stagliano, 1991). Esta línea de investigación solo tiene coherencia teórica si se considera que los intereses de todos los grupos de interés están supeditados al interés del inversor. La contabilidad social y medioambiental, por el contrario, suele tener una concepción más amplia delos destinatarios desuinformación. Gray, Owen y Adams (1996) la definen como el "proceso de comunicar los efectos sociales y medioambientales de la actividad económica de las organizaciones a determinados grupos de interés en la sociedad y a la sociedad en su conjunto. Como tal, implica extender la responsabilidad de las organizaciones (particularmente empresas) más allá del papel tradicional de proporcionar estados financieros a los propietarios del capital (particularmente a los accionistas). Tal extensión se basa en la presuposición de que las empresas tienen responsabilidades más amplias que hacer dinero para los accionistas" (p. 3)

Desde este punto de vista, la pregunta acerca de la relevancia valorativa de la información social y medioambiental en el mercado de valores carece 
de un fundamento sólido, ya que los intereses de los inversores pueden ser independientes, coincidentes o contrarios a los de otros grupos de interés. En determinados casos estos intereses pueden ser coincidentes, en cuyo caso se producirá una situación positiva para todos los grupos de interés. Tal puede ser el caso del ahorro energético que permite ahorrar costes a la propiedad de la empresa, al tiempo que daños medioambientales. Esta situación se conoce en la literatura anglosajona como "business case". Muchos son los que critican el business case porque entienden que ha pasado de ser una posibilidad a un discurso que esconde situaciones de indiferencia o de intereses contrapuestos. Un ejemplo de lo último puede ser la utilización de mano de obra esclava en la cadena de suministro de la empresa que puede abaratar costes, pero que va obviamente en contra de los intereses de las personas que sufren la esclavitud y de los principios de respeto a los derechos humanos de gobiernos y sociedades.

En qué medida domina el "business case" o el conflicto de intereses es una cuestión de perspectiva, pero podemos estar de acuerdo en que es probable que existan ambas situaciones. Gray (2006) se muestra tajante cuando afirma que los informes de sostenibilidad solo son relevantes para el mercado de capitales en la medida en que sean insustanciales desde el punto de vista del desarrollo sostenible.

\section{Teorías descriptivas}

La teoría positiva de la contabilidad ha orientado la atención de la investigación contable hacia la utilización de la contabilidad en procesos de negociación y de asignación de recursos (Monterrey Mayoral, 1998). De este modo, si bien en las perspectivas anteriores el objeto de investigación es principalmente la representación de la realidad económica, la investigación positiva ha cambiado sustancialmente el foco, con una consideración económica de la contabilidad. Podemos decir que en la teoría positiva de la contabilidad la contabilidad ya no representa la economía, sino que es economía.

Con esta perspectiva, la investigación contable centra su atención en las decisiones de los directivos, bajo la premisa de que la información tiene consecuencias económicas para los individuos y las organizaciones, y de que las decisiones informativas están determinadas por una distribución de costes e ingresos posibles de la información.

Estas ideas han florecido en el campo de la contabilidad social y medioambiental, dando lugar a corrientes de investigación inspiradas por la teoría de los grupos de interés y por la teoría de la legitimidad. He englobado estas teorías dentro de la denominación de teorías descriptivas, en el entendido de que estas teorías tratan de caracterizar 
y explicar regularidades empíricas en la emisión de información social y medioambiental.

\section{Teoría de los grupos de interés}

Como ya es conocido, los grupos de interés (stakeholders) se definen como aquellos individuos o grupos que afectan o se ven afectados por la consecución del propósito de la organización (Freeman, 2010). La teoría de los grupos de interés fue formulada para comprender cómo actúan las organizaciones en un entorno caracterizado por intereses diversos. En contabilidad social y medioambiental, esta teoría se aplica a la explicación de las respuestas informativas de la empresa a los diferentes intereses presentes, considerando el efecto de dichas respuestas en los objetivos estratégicos de la empresa y en los objetivos de los propios directivos. Según Deegan y Blomquist (2006), esta teoría sugiere que la revelación de un tipo particular de información se realizará para ganar o mantener el apoyo de determinados grupos de interés, que tienen poder derivado del control de recursos necesarios para la empresa (Islam y Deegan, 2008).

El modelo propuesto por Deegan y sus colegas (entre otros autores) permite la incorporación de recursos no estrictamente económicos y la consideración de grupos de interés de los que, de forma más general, la organización depende para su supervivencia (Ullmann, 1985). Esto permite la incorporación de intereses sociales y medioambientales. Por ejemplo, una empresa minera podría utilizar un determinado tipo de información en respuesta a las demandas de una organización ecologista, si los directivos de la primera perciben que la segunda es un grupo de interés poderoso (controla recursos necesarios para la primera) (Deegan y Blomquist, 2006).

Estudiosos de la estrategia corporativa (Mitchell et al., 1997) han sofisticado el concepto de poder/control de los grupos de interés, para conocer la prominencia de estos colectivos. Para ello propusieron tres atributos de la prominencia de los grupos de interés, incluyendo al igual que Ullmann (1985) y Deegan y Blomquist (2006) el poder, pero añadiendo la legitimidad y la urgencia. Un grupo de interés será más prominente cuanto más legítimo, poderoso y/o urgente sea su interés sobre la organización.

La legitimidad consiste en la validez o la aprobación que recibe por parte de la sociedad, la demanda de un interés específico de un grupo de interés. Así es que los intereses de los grupos serán tanto más legítimos, cuanto más alineados estén con los valores y normas sociales. Por ejemplo, el interés de aquel que propone un soborno no será legítimo, mientras que 
el interés de los grupos que proponen el respeto de los derechos de la infancia sí lo serán en la sociedad actual.

El poder es el grado en el que el individuo o grupo de interés puede persuadir o inducir a otros individuos a actuar conforme con sus intereses. Normalmente, el poder está asociado al control de recursos coercitivos, financieros o simbólicos que son necesarios para la organización. Este atributo de los grupos de interés sería el que está más en consonancia con la perspectiva de Deegan y sus colegas.

La urgencia se refiere a la medida en que los grupos de interés consideran importantes sus intereses $y$, consecuentemente, demandan que éstos sean atendidos urgentemente. Este atributo de la prominencia requiere un poco de más explicación. Mitchell, Agle y Wood (1997) arguyen que la urgencia se refiere a: (a) la importancia de la oportunidad para resolver la reivindicación de los grupos de interés, ya que éstos consideran inaceptable la demora en su cumplimiento; y (b): la importancia que las pretensiones tienen para la relación entre los grupos de interés y la organización. Mitchell et al.. (1997) consideran que la urgencia aporta a los demás atributos un carácter dinámico. Así, por ejemplo, podría considerarse urgente una demanda que se va a plantear o se ha planteado en la Asamblea General de Accionistas o mediante un boicot.

Diferentes estudios han utilizado este marco teórico para explicar las prácticas informativas de las empresas. Así, Neu, Warsame y Pedwell (1998) encontraron que las empresas dan respuesta (en forma de revelación de información ambiental) en mayor medida a los intereses de los grupos de interés financieros y al gobierno, que a los grupos ambientalistas, interpretando que es el poder de los grupos de interés el que determina la respuesta de las organizaciones. Por su parte, Islam y Deegan (2008) hicieron un estudio de la revelación de información social y medioambiental por las principales empresas exportadoras de ropa de Bangladesh, recurriendo tanto al análisis de contenido como a entrevistas semiestructuradascondirectivos, concluyendoquela actividadinformativa de estas empresas se explica principalmente por la prominencia de los grupos de interés occidentales (empresas multinacionales compradoras, consumidores o medios de comunicación), en detrimento de la atención a los grupos de interés locales. Un interesante estudio adicional sobre el mismo sector en Bangladesh es el realizado por Sinkovics, Hoque y Sinkovics (2016). Estos autores observaron cómo las exigencias impuestas (de nuevo) por los grupos de interés occidentales, en forma de auditorías sociales, tienen una influencia capital en el desempeño y la revelación de información de estas organizaciones. La prominencia de los grupos de interés que demandan la realización de estas auditorías hace que éstas 
hayan tenido ciertos efectos, por ejemplo, en la mejora de la seguridad e higiene en el ámbito laboral de estas fábricas. Sin embargo, estos autores encontraron que estas auditorías están basadas en una visión occidental de las relaciones laborales, pero exhiben una falta de entendimiento del contexto cultural y de la pobreza existente en Bangladesh. Estas limitaciones hacen que las auditorías sociales tengan, paradójicamente, consecuencias indeseadas sobre los salarios o la estabilidad laboral, entre otras cuestiones. Como uno de los directivos de estas empresas expresó a los investigadores:

"Las auditorías de cumplimiento son como una ornamentación o decoración de nuestra fábrica. ¿Por qué preferimos comprar el mismo producto en centros comerciales, en lugar de en las tiendas tradicionales? Es debido al mejor ambiente que se crea en los centros comerciales. Las auditorías de cumplimiento también mejoran la belleza de nuestras fábricas o, mejor dicho, el ambiente para cuando las empresas multinacionales van de compras" ( $p$. 643)

\section{Teoría de la legitimidad}

La teoría de la legitimidad también trata de explicar la revelación de información social y medioambiental, considerando que los directivos y sus empresas están motivados por consideraciones económicas y sociales. De acuerdo con esta teoría, las acciones informativas se realizan con el propósito de asegurar de que la sociedad tenga la percepción de que las organizaciones actúan conforme a las reglas sociales. En este caso se considera que las organizaciones son "legítimas" (Islam y Deegan, 2008). Pero las normas sociales y las condiciones en las que se percibe la empresa no son constantes, sino que van cambiando. En estas circunstancias es frecuente que se produzcan brechas de legitimidad, razón por la cual la empresa estará inmersa en un constante proceso de legitimación. La revelación de información social y medioambiental juega un papel en dicho proceso de legitimación, tratando de minimizar el impacto de las brechas de legitimidad (Lindblom, 1993).

En su conocido artículo de 1993, Lindblom propuso una tipología de posibles respuestas de la organización dentro de este proceso de legitimación. La primera respuesta identificada consiste en la revelación de información que puede utilizarse en la comunicación de los cambios que se han efectuado para lograr una consonancia con las expectativas sociales.

Si las organizaciones optan por no cambiar su actuación o sus objetivos, la segunda respuesta descrita por Lindblom (1993) radica en la utilización 
de la revelación de información social y medioambiental para cambiar la percepción que de la organización tienen los públicos externos relevantes. Una parte sustancial de la investigación que ya antes he denominado "crítica funcional" utiliza de forma parcial este argumento para explicar las razones por las que las empresas publican información social y medioambiental que no se corresponde con su desempeño real (Cho y Patten, 2007).

Cuando las organizaciones no cambian su comportamiento ni sus objetivos y tampoco pueden cambiar la percepción que la sociedad tiene de ellas mediante la explicación de su actuación, Lindblom (1993) propuso una tercera categoría para describir la estrategia consistente en que las empresas se embarquen en tratar de asociar su actividad con símbolos que sí tienen legitimidad social.

La última estrategia descrita por esta autora consistiría en manipular la sociedad para que sus expectativas cambien, tratando así de cerrar la brecha de legitimidad mediante el cambio de la sociedad, en lugar de gestionar el cambio de la empresa.

Son numerosos los estudios basados en la teoría de la legitimidad (revisados por Deegan, 2002). Estos estudios han encontrado, por ejemplo, un incremento de información medioambiental publicada por empresas cuya legitimidad podría haberse dañado por catástrofes ambientales (Patten, 1992) o por sanciones ambientales (Deegan y Rankin, 1996). Así, del mismo modo, Cho et al.. (2012) encontraron una correlación negativa entre información medioambiental y mediciones independientes de resultados ambientales.

En mi opinión, la popularidad de esta teoría se debe a la sencillez de sus presupuestos, a su consistencia con las teorías dominantes en contabilidad y a la disponibilidad de evidencia (en forma de memorias o de bases de datos) susceptible de tratamiento estadístico. Esta abundancia de estudios en teoría de la legitimación hace que la posibilidad de realizar una contribución sea más limitada. No obstante, creo que el estudio de la realidad latinoamericana ofrece la oportunidad de realizar estudios que sí realicen aportaciones suficientes.

Hay otros estudios que han tratado de desarrollar de algún modo la teoría de la legitimación, mediante los tipos de legitimidad (moral, cognitiva y estratégica) identificados por Suchman (1995), mediante las respuestas estratégicas a presiones institucionales categorizadas por Oliver (1991) o a través la diferenciación entre legitimidad y estrategias de reputación y de mantenimiento de la imagen (Bebbington, Larrinaga y Moneva, 2008). 


\section{Nuevo institucionalismo sociológico}

Las investigaciones derivadas de las ideas del nuevo institucionalismo sociológico constituyen una interesante evolución de la teoría de la legitimidad. Más que de perspectiva, sería apropiado hablar de perspectivas en plural, pues son muchos los caminos que se abren aquí. Sin embargo, por conectar con la teoría de la legitimidad, comenzaremos con Scott (2008), para quien la legitimidad, en contraste con Suchman por ejemplo, no es un recurso que se pueda poseer (como sugiere la teoría de la legitimidad), sino "una condición que refleja el alineamiento cultural, el amparo normativo o la consonancia con reglas y leyes relevantes" (1995: 45) y que proporciona estabilidad a las organizaciones.

Un aspecto importante del nuevo institucionalismo sociológico es su interés en el estudio de la estabilidad y el isomorfismo de las organizaciones. Es decir, a pesar del énfasis de las perspectivas anteriores en la caracterización y explicación de diferentes respuestas de las organizaciones, lo cierto es que éstas tienden a tener comportamientos muy parecidos entre sí y estos comportamientos no cambian tanto como pudiera esperarse. Un buen ejemplo de ello lo constituyen las grandes empresas que elaboran memorias de sostenibilidad, todos ellas siguiendo guías similares. La pregunta institucional es la explicación de este comportamiento isomorfo y estable.

El nuevo institucionalismo sociológico (DiMaggio y Powell, 1983; Scott, 2008) aporta diferentes explicaciones a la institucionalización: coercitiva, normativa y cognitiva. La explicación coercitiva no plantea excesivas novedades en relación con planteamientos anteriores, pues está basada en la disciplina de los mercados, de la regulación o de grupos de interés poderosos. Una parte de la institucionalización de prácticas de contabilidad social y medioambiental se puede explicar, como sugiere la teoría de los grupos de interés, por la preeminencia de algunos grupos. Sin embargo, la novedad de esta perspectiva radica en que subraya motivos basados en la lógica de lo apropiado y en la construcción social de la realidad (Higgins y Larrinaga, 2014).

Los individuos participan en la organización, pero también en la sociedad, y están expuestos a expectativas "normativas" sobre la forma correcta de actuar en determinadas circunstancias. De este modo, además de por razones coercitivas, es posible que las empresas publiquen memorias sociales y ambientales por la existencia de expectativas sociales. Es posible que estos comportamientos escapen a la racionalidad económica; el comportamiento colectivo de campos organizativos (isomorfismo) tampoco se puede explicar por los valores éticos individuales. El 
origen de los valores transmitidos a través de este pilar normativo puede encontrarse en la historia, en la educación formal o en las redes profesionales (DiMaggio y Powell, 1983).

Finalmente, las instituciones también están basadas en dimensiones cognitivas. Símbolos, significados y reglas son construcciones sociales (Berger y Luckmann, 1966) que, una vez consolidados, ejercen una presión irresistible hacia la estabilidad y la conformidad, porque hacen que la mayoría de las acciones parezcan de sentido común o, por el contrario, inconcebibles. Estas estructuras cognitivas "forman un respaldo, culturalmente fundamentado y moralmente correcto, de la legitimidad que está fuera de toda cuestión" (Hoffman, 1999) y pueden tener un papel importante en la explicación de la divulgación de información social y medioambiental, aunque es más difícil de explorar empíricamente (Higgins y Larrinaga, 2014) que las dimensiones coercitivas y normativas.

El concepto de institución y de estabilidad es así mismo útil para la explicación de la captura gerencial de la contabilidad social y medioambiental (O'Dwyer, 2003). En el campo del neoinstitucionalismo sociológico, Meyer y Rowan (1977) proponen que los intentos de controlar y coordinar actividades en las organizaciones conducen a conflictos y pérdida de legitimidad $y$, para evitarlo, los elementos estructurales se desacoplan de las actividades (Covaleski y Dirsmith, 1986). La literatura en contabilidad social (Adams, 2004; O'Dwyer y Unerman, 2007) es rica en ejemplos de estructuras de desacoplamiento entre la responsabilidad y las actividades organizacionales.

De modo similar a la noción desacoplamiento, Cho et al.. (2015) han estudiado cómo, en situaciones de presiones sociales e institucionales contradictorias, las organizaciones utilizan sus informes sociales y medioambientales - dicho en sus propios términos- como "hipocresía organizada", de forma que creen una fachada simbólica que proporciona una apariencia de legitimidad. Aunque Cho y sus colegas recurren a la explicación finalista de la satisfacción de los grupos de interés, la explicación institucional no acentúa tanto esta cuestión como la necesidad del desacoplamiento en situaciones de demandas conflictivas para la estabilidad organizativa.

\section{Conclusiones}

En este trabajo he repasado algunas perspectivas de investigación en contabilidad social y medioambiental, dividiéndola en dos apartados. En primer lugar, he revisado las perspectivas más convencionales, sus inicios normativos, la investigación sobre el marco conceptual y el paradigma de utilidad. Todas estas perspectivas tienen todavía vigencia en temas de 
investigación en contabilidad social y medioambiental que siguen siendo actuales.

En segundo lugar, he revisado el interés de las teorías descriptivas en la explicación de las decisiones informativas de los directivos. Esta investigación trata de responder a la pregunta de por qué las empresas revelan información social y medioambiental. La teoría de los grupos de interés, la teoría de la legitimidad y la teoría institucional son tres marcos de referencia habituales en esta literatura.

En esta discusión resulta relevante un aspecto metodológico básico para poner en perspectiva las diferentes aproximaciones teóricas examinadas: la distinción entre prescripción y descripción. Las teorías descriptivas tratan de caracterizar y explicar regularidades observadas en la emisión de información social y medioambiental por parte de las empresas. Tanto la teoría de los grupos de interés, como la teoría de la legitimidad, han venido a concluir que las decisiones informativas de los directivos responden a los intereses y preeminencia de los grupos de interés o a la consistencia entre actuación percibida de la empresa y valores sociales. De este modo, cuando Cho et al. (2012) encuentran que las empresas con peores resultados medioambientales son las que están proporcionando más información medioambiental y que a más información, mejor reputación de la empresa, estos autores están simplemente caracterizando el comportamiento de las empresas de acuerdo con fuentes de evidencia derivadas de los informes de las empresas y fuentes externas a las empresas, pero de ningún modo están prescribiendo un comportamiento. Es decir, Cho et al. (2012) no recomiendan a las malas empresas que divulguen más información para "hacerse perdonar sus pecados" (prescripción), simplemente están describiendo el comportamiento de las empresas y explicándolo de acuerdo con la teoría de la legitimidad. De hecho, a veces es necesario leer entre líneas estos artículos descriptivos para entender su mensaje principal, que es coincidente con lo que anteriormente llamé la crítica funcional de la contabilidad social y medioambiental.

Finalmente, quiero reseñar una limitación muy importante de este trabajo. Al examinar las líneas de investigación que están más consolidadas en contabilidad social y medioambiental, la revisión ha dejado fuera las perspectivas más constructivistas, interdisciplinares o críticas. Creo que la comprensión de la contabilidad social y medioambiental demanda transitar por las perspectivas más consolidadas, pero son las perspectivas alternativas las que tienen un futuro más prometedor. 


\section{Referencias Bibliográficas}

- ADAMS, Carol A. (2004), "The ethical, social and environmental reportingperformance portrayal gap". En: Accounting, Auditing \& Accountability Journal, Vol. 17 N¹5. Bingley (UK): MCB University Press. Pp. 731-757.

- $\quad$ ANTHEAUME, Nicolás. (2004), "Valuing external costs. From theory to practice: implications for full cost environmental accounting". En: European Accounting Review, Vol. 13 № 3. Abingdon (UK): Taylor \& Francis. Pp. 443-464.

- BALL, Ray y BROWN, Philip. (1968). "An Empirical Evaluation of Accounting Income Numbers". En: Journal of Accounting Research, Vol. 6 N $^{\circ} 2$. Chicago: The Accounting Research Center- University of Chicago. Pp. 159-178.

- BEBBINGTON, Jan y GRAY, Robert (2001), "An Account of Sustainability: Failure, Success and a Reconceptualization". En: Critical Perspectives on Accounting, Vol. 12 № 5. Cambridge: Academic Press. Pp. 557-588.

- BEBBINGTON, Jan y LARRINAGA, Carlos (2014), "Accounting and sustainable development: An exploration": En: Accounting, Organizations and Society, Vol. $39 \mathrm{~N}^{\circ}$ 6. Oxford (UK): Pergamon Press. Pp. 395-413.

- BEBBINGTON, Jan, LARRINAGA, Carlos y MONEVA, José María (2008), “Corporate social reporting and reputation risk management". En: Accounting, Auditing \& Accountability Journal, Vol. $21 \mathrm{~N}^{\circ} 3$. Bingley (UK): MCB University Press. Pp. 337361.

- $\quad$ BERGER, Peter y LUCKMANN, Thomas. (1966), The social construction of reality. Buenos Aires: Amorrortu Editores.

- BOIRAL, Oliver. (2013), "Sustainability reports as simulacra? A counter-account of A and A+ GRI reports". En: Accounting, Auditing and Accountability Journal, Vol. $26 N^{\circ}$ 7. Bingley (UK): MCB University Press. Pp. 1036-1071.

- CORMIER, Denis, MAGNAN, Michel y MORARD, Bernard. (1993), "The impact of corporate pollution on market valuation: some empirical evidence". En: Ecological Economics, Vol. $8 \mathrm{~N}^{\circ}$ 2. Amsterdam: International Society for Ecological Economics (ISEE). Pp. 135-155.

- COVALESKI, Mark A.y DIRSMITH, Mark W. (1986), "The budgetary process of power and politics". En: Accounting, Organizations and Society, Vol. $11 \mathrm{~N}^{\circ} 3$. Oxford (UK): Pergamon Press. Pp. 193-214.

- $\quad \mathrm{CHO}$, Charles H., GUIDRY, Ronald P., HAGEMAN, Amy M. y PATTEN, Dennis M. (2012), "Do actions speak louder than words? An empirical investigation of corporate environmental reputation" En: Accounting, Organizations and Society, Vol. $37 \mathrm{~N}^{\circ}$ 1. Oxford (UK): Pergamon Press. Pp. 14-25.

- $\quad$ CHO, Charles H., LAINE, Matías, ROBERTS, Robin W. y RODRIGUE, Michelle. (2015), "Organized hypocrisy, organizational façades, and sustainability reporting". En: Accounting, Organizations and Society, Vol. $40 \mathrm{~N}^{\circ}$ Supplement C. Oxford (UK): Pergamon Press. Pp. 78-94.

- CHO, Charles H. y PATTEN, Dennis M. (2007), "The role of environmental disclosures as tools of legitimacy: A research note". En: Accounting, Organizations and Society, Vol. 32 N 7-8. Oxford (UK): Pergamon Press. Pp. 639-647.

- DEEGAN, Craig. (2002), "The legitimising effect of social and environmental disclosures - a theoretical foundation". En: Accounting, Auditing \& Accountability Journal, Vol. $15 \mathrm{~N}^{\circ}$ 3. Bingley (UK): MCB University Press. Pp. 282-311. 
- DEEGAN, Craig y BLOMQUIST, Christopher. (2006), "Stakeholder influence on corporate reporting: An exploration of the interaction between WWF-Australia and the Australian minerals industry". En: Accounting, Organizations and Society, Vol. $31 \mathrm{~N}^{\circ}$ 4-5. Oxford (UK): Pergamon Press. Pp. 343-372.

- DEEGAN, Craig y RANKIN, Michaela. (1996), "Do Australian companies report environmental news objectively? An analysis of environmental disclosures by firms prosecuted successfully by the Environmental Protection Authority". En: Accounting, Auditing \& Accountability Journal, Vol. $9 \mathrm{~N}^{\circ}$ 2. Bingley (UK): MCB University Press. Pp. 50-67.

- DEEGAN, Craig y UNERMAN, Jeffrey. (2011), Financial Accounting Theory. Londres: McGraw-Hill.

- DIMAGGIO, Paul J. y POWELL, Walter W. (1983), "The iron cage revisited institutional isomorphism and collective rationality in organizational fields". En: American Sociological Review, Vol. 48 N² 2. Washington, D.C.: American Sociological Association. Pp. 147-160.

- EHNERT, Ina, PARSA, Sepideh, ROPER, Ian, WAGNER, Marcus y MULLER-CAMEN, Michael. (2016), "Reporting on sustainability and HRM: a comparative study of sustainability reporting practices by the world's largest companies". En: The International Journal of Human Resource Management, Vol. $27 \mathrm{~N}^{\circ} 1$. Abingdon (UK): Taylor \& Francis. Pp. 88-108.

- ESTES, Ralph W. (1972), "Socio-Economic Accounting and External Diseconomies". En: The Accounting Review, Vol. $47 \mathrm{~N}^{\circ}$ 2. Lakewood Ranch (USA): American Accounting Association. Pp. 284-290.

- FEE. (2000), "Towards a General Accounting Framework for Environmental Reporting". Brussels: Féderation des Experts Comptables Européens.

- FREEDMAN, Martin y STAGLIANO, A. J. (1991), "Differences in Social-Cost Disclosures: A Market Test of Investor Reactions". En: Accounting, Auditing \& Accountability Journal, Vol. $4 \mathrm{~N}^{\circ} 1$. Bingley (UK): MCB University Press. Pp. 294290.

- FREEMAN, Edward. (2010), Strategic management: A stakeholder approach. Cambridge: (UK): Cambridge University Press.

- GÓMEZ-VILLEGAS, Mauricio. y OSPINA-ZAPATA, Carlos Mario (2009), Avances Interdisciplinarios para una Comprensión Crítica de la Contabilidad. Textos Paradigmáticos de las Corrientes Heterodoxas. Bogotá: Universidad Nacional de Colombia.

- GRAY, Rob. (1992), "Accounting and environmentalism: An exploration of the challenge of gently accounting for accountability, transparency and sustainability". En: Accounting, Organizations and Society, Vol. $17 \mathrm{~N}^{\circ}$ 5. Oxford (UK): Pergamon Press. Pp. 399-425.

- GRAY, Rob. (2002), "The social accounting project and Accounting Organizations and Society Privileging engagement, imaginings, new accountings and pragmatism over critique?". En: Accounting, Organizations and Society, Vol. $27 \mathrm{~N}^{\circ}$ 7. Oxford (UK): Pergamon Press. Pp. 687-708.

- GRAY, Rob. (2006), "Social, environmental and sustainability reporting and organisational value creation?: Whose value? Whose creation?". En: Accounting, Auditing \& Accountability Journal, Vol. $19 \mathrm{~N}^{\circ}$ 6. Bingley (UK): MCB University Press. Pp. 793-819

- GRAY, Rob. (2010), "Is accounting for sustainability actually accounting for sustainability.and how would we know?. En: An exploration of narratives of 
organisations and the planet", Accounting, Organizations and Society, Vol. $35 \mathrm{~N}^{\circ}$ 1. Oxford: Pergamon Press . Pp. 47-62.

- $\quad$ GRAY, Rob., OWEN, Dave. y MAUNDERS, Keith. (1987), Corporate social reporting: accountaing and accountability. Londres: Prentice-Hall International.

- GRAY, Rob., OWEN, Dave and ADAMS, Carol A. (1996), Accounting and accountability: Changes and challenges in corporate social and environmental reporting. Londres: Prentice Hall, London.

- GRI. (2016), "GRI Sustainability Reporting Standards 2016". Amsterdam: Global Reporting Initiative.

- HAHN, Rüdiger y KÜHNEN, Michael. (2013), "Determinants of sustainability reporting: a review of results, trends, theory, and opportunities in an expanding field of research". En: Journal of Cleaner Production, Vol. $59 \mathrm{~N}^{\circ} 0$. Amsterdam: Elsevier. Pp. 5-21.

- HIGGINS, Colin y LARRINAGA, Carlos. (2014), "Sustainability reporting: insights from neo-institutional theory". En: Unerman, J., O'Dwyer, B. y Bebbington, J. (Eds.), Sustainability accounting and accountability, 2a. ed. Oxford (UK): RoutledgeOxon.

- HOFFMAN, Andrew J. (1999), "Institutional evolution and change: Environmentalism and the US chemical industry". En: Academy of Management Journal, Vol. $42 \mathrm{~N}^{\circ}$ 4. Nueva York: Academy of Management-AOM. Pp. 351-371.

- ISLAM, Muhammad Azizuly DEEGAN,Craig.(2008),"Motivationsforanorganisation within a developing country to report social responsibility information: Evidence from Bangladesh". En: Accounting, Auditing \& Accountability Journal, Vol. 21 N $^{\circ} 6$. Bingley (UK): MCB University Press. Pp. 850-874.

- JUNIOR, Renzo Mori, BEST, Peter J. y COTTER, Julie. (2014), "Sustainability Reporting and Assurance: A Historical Analysis on a World-Wide Phenomenon". En: Journal of Business Ethics, Vol. 120 № 1. Berlín: Springer. Pp. 1-11.

- $\quad$ KPMG. (2017), “KPMG Survey of Corporate Responsibility Reporting 2017 “. En: KPMG: www.kpmg.com/sustainability.

- LARRINAGA, Carlos. (1999), "Perspectivas alternativas de investigación en contabilidad: Una revisión". En: Revista de Contabilidad, Vol. 2 N $^{\circ} 3$. Madrid: ASEPUC. Pp. 103-132.

- LINDBLOM, Cristi K. (1994), "The implications of organizational legitimacy for corporate social performance and disclosure". En: Critical Perspectives on Accounting Conference, New York.

- MEYER, John W. y ROWAN, Brian. (1977), "Institutionalized organizations - formalstructure as myth and ceremony". En: American Journal of Sociology, Vol. $83 \mathrm{~N}^{\circ} 2$. Chicago: Universidad de Chicago. Pp. 340-363.

- MITCHELL, Ronald K., AGLE, Bradley R. y WOOD, Donna J. (1997), "Toward a Theory of Stakeholder Identification and Salience: Defining the Principle of Who and What Really Counts". En: The Academy of Management Review, Vol. $22 \mathrm{~N}^{\circ} 4$. Nueva York: Academy of Management-AOM. Pp. 853-886.

- MONTERREY-MAYORAL, Juan. (1998), "Un recorrido por la contabilidad positiva”. En: Revista Española de Financiación y Contabilidad, Vol. 27 № 95. Madrid: Asociación Española de Contabilidad y Adminsitración de Empresas-AECA. Pp. 427-467. 
- NEU, Dean, WARSAME, Alí Haji y PEDWELL, Katie. (1998), “Managing public impressions: Environmental disclosures in annual reports". En: Accounting, Organizations and Society, Vol. 23 N 3. Oxford (UK): Pergamon Press. Pp. 265-282.

- O'DWYER, Brendan y UNERMAN, Jeffrey. (2007), "From functional to social accountability: Transforming the accountability relationship between funders and non-governmental development organisations". En: Accounting, Auditing \& Accountability Journal, Vol. $20 \mathrm{~N}^{\circ}$ 3. Bingley (UK): MCB University Press. Pp. 446471.

- O'DWYER, Brendan. (2003), "Conceptions of corporate social responsibility: the nature of managerial capture". En: Accounting, Auditing \& Accountability Journal, Vol. $16 \mathrm{~N}^{\circ}$ 4. Bingley (UK): MCB University Press. Pp. 523-557.

- OLIVER, Christine. (1991), "Strategic responses to institutional processes". En: Academy of Management Review, Vol. $16 \mathrm{~N}^{\circ}$ 1. Nueva York: Academy of Management-AOM. Pp. 145-179.

- $\quad$ PATTEN, Dennis M. (1992), "Intra-industry environmental disclosures in response to the Alaskan oil spill: A note on legitimacy theory". En: Accounting, Organizations and Society, Vol. $17 \mathrm{~N}^{\circ}$ 5. Oxford (UK): Pergamon Press. Pp. 471-475.

- PINA-MARTíNEZ, Vicente. (1991), "Investigacion empirica y normalizacion contable". En: Revista Española de Financiación y Contabilidad, Vol. 20 № 66. Madrid: Asociación Española de Contabilidad y Adminsitración de EmpresasAECA. Pp. 83-126.

- RAMANATHAN, Kavasseri V. (1976), "Toward a Theory of Corporate Social Accounting". En: The Accounting Review, Vol. 51 N$^{\circ}$ 3. Lakewood Ranch (USA): American Accounting Association. Pp. 516-528.

- $\quad$ RIAHI-BELKAOUI, Ahmed. (2004), Accounting theory. Londres: Academic Press.

- $\quad$ SCOTT, W. Richard. (2008). Institutions and organizations. Newcastle (UK): Sage.

- $\quad$ SINKOVICS, Noemi, HOQUE, Samia Ferdous and SINKOVICS, Rudolf R. (2016), “Rana Plaza collapse aftermath: are CSR compliance and auditing pressures effective?". En: Accounting, Auditing \& Accountability Journal, Vol. $29 \mathrm{~N}^{\circ}$ 4. Bingley (UK): MCB University Press. Pp. 617-649.

- SPICER, Barry H. (1978), "Investors, Corporate Social Performance and Information Disclosure: An Empirical Study". En:The Accounting Review, Vol. 53 NN$^{\circ}$. Lakewood Ranch (USA): American Accounting Association. Pp. 94-111.

- $\quad$ SUCHMAN, Mark C. (1995), "Managing Legitimacy: Strategic and Institutional Approaches". En: Academy of Management Review, Vol. 20 NN$^{\circ}$ 3. Nueva York: Academy of Management-AOM. Pp. 571-610.

- $\quad$ TROTMAN, Ken T. y BRADLEY, Graham W. (1981), "Associations between social responsibility disclosure and characteristics of companies". En: Accounting, Organizations and Society, Vol. 6 N ${ }^{\circ}$ 4. Oxford (UK): Pergamon Press. Pp. 355-362.

- ULLMANN, Arieh A. (1985), "Data in search of a theory - a critical-examination of the relationships among social performance, social disclosure, and economicperformance of United-States firms"En: Academy of Management Review, Vol. 10 $N^{\circ}$ 3. Nueva York: Academy of Management-AOM. Pp. 540-557.

\begin{tabular}{c|l|}
$\begin{array}{c}\text { Para citar } \\
\text { este artículo: }\end{array}$ & $\begin{array}{l}\text { Larrinaga, C. (2017). En torno a la evolución de la } \\
\text { investigación en Contabilidad Social y Medioambiental. } \\
\text { Teuken Bidikay Vol. 08 N 11. Pp. 21-38. }\end{array}$ \\
\hline
\end{tabular}

